\title{
Enquête sur la consommation des produits laitiers dans le milieu socio-professionnel de la recherche et de l'enseignement supérieur
}

\author{
par \\ Jean ADRIAN \\ C.N.R.S., 9, rue J.-Hetzel - 92190 Bellevue \\ «Mesurer, c'est limiter, \\ et, bien souvent, c'est méconnaître ". \\ F. X. СНАВОСНЕ.
}

\section{INTRODUCTION}

Les besoins nutritionnels et les habitudes alimentaires subissent une évolution importante durant le $\mathrm{xx}^{\mathrm{e}}$ siècle, conséquence directe des transformations profondes des modalités d'existence, des progrès d'ordre technologique et des modes de commercialisation des denrées alimentaires.

Le phénomène le plus connu est une réduction de la consommation de certaines productions alimentaires «pauvres » et principalement énergétiques comme le pain, les légumes secs, les pommes de terre et un accroissement de certains produits plus « flatteurs » ou plus " nobles » comme la viande, les biscottes et les biscuits, les boissons non-alcoolisées (eaux minérales, boissons gazeuses).

Dans ce contexte, la consommation des produits laitiers subit une évolution assez ambiguë, sur laquelle les spécialistes s'interrogent. Notamment, dans de nombreux pays industrialisés, la consommation de lait paraît avoir atteint un maximum et tend même plutôt à décliner, tandis que l'attrait pour les fromages fermentés et les desserts lactés croît de façon importante et continue. Rien n'indique que ces évolutions doivent se modifier dans un avenir proche.

La table 1 situe l'évolution de la consommation apparente de quelques produits alimentaires de base, produits laitiers et céréa- 


\section{$T A B L E 1$}

Consommation apparente des produits alimentaires en France et perspectives (1) (en g par tête et par jour)

\begin{tabular}{l|c|c|c}
\hline & 1960 & 1970 & 1980 \\
\cline { 2 - 3 } & 254 & 252 & 240 \\
Lait frais & 4,4 & 6,3 & 6,85 \\
Lait condensé en poudre & 1,78 & 1,98 & 1,98 \\
Crème fraîche & 33,1 & 23,6 & 44,5 \\
Yoghourts et laits gélifiés & 281 & 37,8 & 49,5 \\
Fromages & 4,1 & 221 & 176 \\
Pain & 10,5 & 5,7 & 8,0 \\
Biscottes & 0,6 & 15,2 & 22,2 \\
Biscuits, pain d'épices & & 1,0 & 1,6 \\
Entremets et desserts instantanés & & & \\
\hline
\end{tabular}

(1) A. Fouquet, rapport M 54, I.N.S.E.E., mai 1976.

liers, mettant en relief l'abandon progressif de certaines formes " simples " au profit de nouvelles présentations plus " élaborées ».

Nous avons voulu préciser la fréquence de consommation des divers produits laitiers en procédant à une enquête dans un milieu socio-professionnel très limité et, peut-être, un peu marginal par rapport à l'ensemble des consommateurs : il s'agit de celui de la recherche - publique et privée - et de l'enseignement supérieur, les spécialistes en matière de nutrition étant exclus de cette enquête.

Les personnes composant ce milieu présentent certaines caractéristiques : elles vivent pour la plupart en milieu urbanisé et leurs achats se font donc pour une part appréciable en faisant appel aux nouveaux circuits de distribution.

Par leur niveau culturel et leur activité professionnelle, elles peuvent être considérées comme averties des problèmes de l'alimentation. Ce dernier point offre une grande importance pour notre projet car il conditionne la participation à l'enquête ainsi que le sérieux et la validité des réponses obtenues. 


\section{MODALITES PRATIQUES}

Nous avons établi un questionnaire se composant de nombreuses questions relatives à la consommation des produits laitiers. Il est prévu pour une à cinq ou six personnes de manière à recueillir les réponses d'un groupe familial entier. Il est accompagné d'une feuille explicative exposant les buts de l'opération, le tout étant envoyé à un certain nombre de centres de recherches et d'établissement d'enseignement supérieur.

Le questionnaire porte sur les points suivants :

1. Age et sexe des personnes enquêtées.

2. Consommez-vous régulièrement du lait liquide entier, demi-écrémé ou écrémé : non, moins de $1 / 4 \mathrm{l} / \mathrm{j}$, environ $1 / 4 \mathrm{l} / \mathrm{j}$, entre $1 / 4$ et $1 / 2$ $1 / \mathrm{j}, 1 / 2 \mathrm{l} / \mathrm{j}$, entre $1 / 2$ et $11 / \mathrm{j}$, plus de $1 \mathrm{l} / \mathrm{j}$ ?

3 Consommez-vous régulièrement du lait condensé ou en poudre, entier ou écrémé : non, oui ?

4. Consommez-vous régulièrement des yoghourts, entiers ou maigres : non, environ $1 \mathrm{j}$ sur 2 , un yoghourt par jour, deux yoghourts par jour, plus de deux yoghourts par jour?

5. Consommez-vous régulièrement des fromages frais (fromages blancs) entiers ou maigres : non, environ $1 \mathrm{j}$ sur 2 , une fois par jour, deux fois par jour?

6. Consommez-vous régulièrement des fromages fermentés :

a) non, environ $1 \mathrm{j}$ sur 2 , une fois par jour, deux fois par jour ?

b) avec une préférence pour le type : Camembert, Gruyère, Bleu ?

7. Evitez-vous de consommer des produits laitiers gras pour ne pas grossir?

8. Evitez-vous de consommer des produits laitiers pour des raisons de " goût » ou de "saveurs » désagréables : lait pasteurisé, lait de longue conservation (UHT), yoghourts, fromages frais, fromages fermentés?

9. En dehors des motifs précédents évitez-vous de consommer des produits laitiers pour des raisons diverses, digestion ou autres : lait liquide, lait en poudre, yoghourts, fromages frais, fromages fermentés?

10. Consommez-vous régulièrement du pain ou des biscottes, ou des produits analogues :

a) non, environ $125 \mathrm{~g} / \mathrm{j}$ (une demi-baguette), entre 125 et $250 \mathrm{~g} / \mathrm{j}$, environ $250 \mathrm{~g} / \mathrm{j}$, plus de $250 \mathrm{~g} / \mathrm{j}$ ?

b) sous forme de pain blanc, pain complet, biscottes ?

11. Parmi vos ascendants, certains ont-ils ou ont-ils eu la réputation de refuser de consommer : du lait, des fromages ? 
Nous sommes conscients qu'en procédant à une enquête de ce type, surgissent deux difficultés :

- Ne sont enregistrées que les réponses des personnes qui acceptent de répondre au questionnaire, ce qui entraîne une certaine sélection au sein de la population qui reçoit les questionnaires. Les réponses des personnes volontaires ne reflètent pas obligatoirement les consommations de l'ensemble d'une population.

- Les personnes ayant accepté de répondre peuvent, de bonne foi, fournir des estimations inexactes.

Ces reproches sont inhérents à cette façon de procéder. C'est pour en réduire la portée que nous nous sommes adressé à un milieu particulièrement apte à participer à ce type d'enquête. De plus, la proportion très élevée de réponses (274 questionnaires remplis sur 600) confère aux résultats une probabilité d'autant plus forte d'être représentatifs du milieu concerné.

Les réponses obtenues proviennent : du Centre de Recherches Rhône-Poulenc à la Croix-de-Berny (117 questionnaires), des Centres de Douai et de Massy de l'ENSIA (60 questionnaires), du Centre de Grignon de l'INA Paris-Grignon (53 questionnaires), de 1'Université de Bordeaux I (29 questionnaires) et du POVAR (CNRS, Bellevue) (15 questionnaires).

Dans l'ensemble elles concernent des personnes localisées dans le Bassin parisien, dont les consommations de produits laitiers ne sauraient être extrapolées sans précautions à l'ensemble de la population française.

\section{POPULATION SUR LAQUELLE A PORTE L'ENQUETE ET CATEGORIES DE CONSOMMATEURS}

Nous disposons de 271 questionnaires exploitables, ce qui correspond au comportement alimentaire de 779 personnes (tab. 2). Il s'agit, en majeure partie de "familles » (questionnaires concernant trois personnes ou plus). Les " couples " (questionnaires où figurent les réponses de deux personnes retenues dans l'enquête) et les « célibataires » (une seule personne retenue) sont nettement moins nombreux. Ce dernier groupe ne doit pas être pris au sens strict c'est-à-dire de personne vivant seule : dans certains cas, des personnes vivant en famille n'ont apporté de réponses que pour ellesmêmes et non pour l'ensemble de leur groupe familial. Cette situation est peut-être assez exceptionnelle.

Les personnes enquêtées sont classées en différentes catégories, en fonction de leur âge et de leur sexe. Il convient préalablement de préciser que le cas des personnes âgées (plus de 70 ans) a été examiné 
TABLE 2. - Personnes ayant répondu à l'enquête

Répartition des groupes familiaux :

Questionnaires reçus

Questionnaires exploitables

Questionnaire relatif à :

1 personne des catégories $\mathbf{A}$ à $\mathbf{M}$ (1)

2 personnes des catégories $\mathrm{A}$ à $\mathrm{M}(2)$

3 personnes ou plus des catégories A à $\mathrm{M}$ (3)

\begin{tabular}{|c|c}
$\begin{array}{c}\text { Nombre de } \\
\text { questionnaires }\end{array}$ & $\begin{array}{c}\text { Nombre de } \\
\text { personnes }\end{array}$ \\
\hline & \\
\hline 274 & 791 \\
271 & 779 \\
70 & 70 \\
59 & 118 \\
138 & 552
\end{tabular}

Répartition par catégorie :

Personnes âgées (71 ans et plus)

A - Hommes (46 à 70 ans)

B - Femmes (46 à 70 ans)

C - Hommes (22 à 45 ans)

D - Femmes (22 à 45 ans)

E - Pré-adultes garçons (16 à 21 ans)

$\mathrm{H}$ - Pré-adultes filles (16 à 21 ans)

$\mathrm{J}$ - Adolescents (12 à 15 ans)

L - Enfants (6 à 11 ans)

$\mathrm{M}$ - Pré-scolaires (3 à 5 ans)

Tout-petits

Total général

Total des catégories retenues ( $\mathrm{A}$ à $\mathbf{M}$ )

Total des adultes (A à $\mathrm{D}$ )

Total des jeunes (E à $M)$

(1) dénommés " célibataires " par simplification.

(2) dénommés " couple » par simplification.

(3) dénommés " famille " par simplification.

à part et que celui des jeunes enfants (en dessous de 3 ans) n'a pas été retenu. Ces décisions ont été prises d'abord en raison du faible nombre de réponses concernant ces personnes. De plus, les personnes âgées ne répondent plus exactement à la notion de groupe socioprofessionnel ; quant aux jeunes enfants, leur alimentation dépend davantage des conseils du pédiatre, des choix de la nourrice et de la mère que de celui de l'enfant lui-même ; ses consommations réelles ne sont même pas toujours parfaitement connues des parents.

Les personnes retenues représentent donc un éventail allant de 3 à 70 ans. Les adultes sont groupés en deux classes en fonction de leur âge : les catégories A et B retiennent les hommes et les femmes de 46 à 70 ans; les catégories $C$ et $\mathrm{D}$ les personnes de 22 à 45 ans. 
Les adultes constituent 67,5 p. 100 de la population enquêtée.

Les jeunes sont subdivisés en un certain nombre de catégories : les pré-adultes, garçons et filles ( $\mathrm{E}$ et $\mathrm{H})$, de 16 à 21 ans dont la croissance est apparemment terminée mais dont le développement physiologique n'est pas achevé pour autant. Les adolescents (catégorie J) correspondent sensiblement au premier cycle de l'enseignement secondaire (12 à 15 ans), les enfants ont entre 6 et 11 ans et représentent la tranche d'âge qui fréquente l'école primaire (catégorie $\mathrm{L}$ ) et enfin les pré-scolaires, de 3 à 5 ans (catégorie $\mathrm{M}$ ). Pour ces trois dernières catégories il n'a pas été établi de sous-groupes en fonction du sexe.

Les jeunes constituent 32,5 p. 100 de la population.

\section{CONSOMMATION DE LAIT}

En raison du caractère urbanisé de la population, pratiquement toutes les formes de lait disponibles ont subi un traitement industriel de stabilisation (pasteurisation, stérilisation) ou de transformation (concentration, séchage). La consommation de lait pris directement à la production ou commercialisé selon des critères de haute qualité bactériologique doit représenter un cas presque exceptionnel pour les personnes ayant répondu à l'enquête.

La table 3 montre que d'une manière générale les préférences vont vers le lait entier, le demi-écrémé n'étant consommé que par 1/3 des individus et le lait entièrement écrémé n'occupant qu'une très

\section{TABLE 3}

Consommation des produits laitiers en fonction de leur taux de matières grasses (réponse facultative)

\begin{tabular}{|c|c|c|c|c|}
\hline & \multirow{2}{*}{$\begin{array}{l}\text { Nombre de } \\
\text { réponses }\end{array}$} & \multicolumn{3}{|c|}{ Nature du produit } \\
\hline & & entier & demi-écrémé & maigre \\
\hline Lait liquide & 146 & 59,5 p. 100 & 33,5 p. 100 & 7,0 p. 100 \\
\hline Lait condensé et sec & 32 & 40,5 p. 100 & & 59,5 p. 100 \\
\hline Yoghourts & 103 & 85,5 p. 100 & & 14,5 p. 100 \\
\hline Fromages frais & 55 & 78,0 p. 100 & & 22,0 p. 100 \\
\hline
\end{tabular}


modeste place. Par contre, à l'état de poudre, le lait écrémé semble d'un usage plus répandu, d'autant qu'on puisse en juger par des réponses ayant un caractère facultatif.

La consommation de lait liquide décroît de façon marquée et régulière avec l'âge du consommateur. Entre 3 et 5 ans (catégorie M) 97 p. 100 des jeunes consomment du lait liquide, entre 16 et 21 ans $(\mathrm{E}$ et $\mathrm{H})$ ce pourcentage tombe à $82 \mathrm{p} .100$ et il n'existe plus que 52 p. 100 de consommateurs pour la tranche d'âge 46-70 ans (A et B) (tab. 4).

Le phénomène est indépendant du sexe, une évolution identique s'observe chez les hommes comme chez les femmes.

Cette régression apparaît également à l'examen des quantités consommées : le plus grand nombre d'adultes consomment moins de $1 / 4$ de litre par jour tandis que chez les jeunes la consommation la plus fréquente est de $1 / 4$ de litre.

Ce phénomène n'est qu'à peine corrigé par la consommation des laits transformés. Si 15 à 50 p. 100 des personnes font appel à du lait condensé ou en poudre, la plupart d'entre elles utilisent déjà du lait liquide.

Ainsi, seulement 4 à 9 p. 100 des adultes utilisent des laits transformés à l'exclusion de lait liquide.

$\mathrm{Au}$ total, selon les catégories, 32,5 à 39,5 p. 100 des adultes (A à D) ne consomment aucune forme de lait tandis que dans les catégories de jeunes les non-consommateurs représentent de 9 à 17 p. 100, exception faite des tout-petits de 3 à 5 ans (M.).

Sur 740 personnes, 261 ne reçoivent pas de lait liquide $(32,25$ p. 100) et 227 n'ont aucun apport de lait, sous aucune forme que ce soit $(30,7$ p. 100).

\section{LES REFUS DE CONSOMMATION DE LAIT}

Pour quelles raisons 30 p. 100 des personnes ne font-elles pas appel au lait dans leur alimentation ? La question offre d'autant plus d'intérêt que le contenu nutritionnel du lait est tout à fait remarquable et que son rapport "valeur alimentaire/prix " est très probablement le plus élevé de toutes les productions agricoles.

Quelques remarques peuvent être avancées au vu des résultats enregistrés et de quelques notations ajoutées aux questionnaires :

a) Il peut se manifester une certaine méficnce vis-à-vis d'un produit apparaissant comme "industriel » aux yeux de certains consommateurs, par opposition à la production prise directement à la ferme. Cette position a été exprimée avec plus ou moins de vigueur dans quelques questionnaires. Elle va à l'encontre de la consommation de 
TABLE 4. - Consommation des laits en fonction de la catégorie.

\begin{tabular}{|c|c|c|c|c|c|c|c|c|c|}
\hline & \multicolumn{9}{|c|}{ Catégorie } \\
\hline & $\mathrm{A}$ & B & $\mathrm{C}$ & $\mathrm{D}$ & $\mathrm{E}$ & $\mathrm{H}$ & $\mathbf{J}$ & $\mathrm{L}$ & M \\
\hline \multicolumn{10}{|l|}{ Lait liquide } \\
\hline Pas de consommation (p. 100) & 48,5 & 47,5 & 37,0 & 39,5 & 20,0 & 16,5 & 19,0 & 12,5 & 3,0 \\
\hline Moins de $1 / 41$ (p. 100) & 30,0 & 29,0 & 30,5 & 27,0 & 23,0 & 30,0 & 17,0 & 13,5 & 14,5 \\
\hline Environ $1 / 41$ (p. 100$)$ & 15,5 & 14,5 & 19,0 & 23,0 & 26,0 & 33,0 & 36,0 & 40,5 & 44,0 \\
\hline $1 / 4$ à $1 / 2 \mathrm{l}($ p. 100$)$ & 3,5 & 5,5 & 6,5 & 5,0 & 14,5 & 3,5 & 15,0 & 24,0 & 23,5 \\
\hline $1 / 21$ (p. 100$)$ & 1,0 & 2,5 & 4,5 & 4,0 & 14,5 & 3,5 & 4,5 & 5,5 & 3,0 \\
\hline $1 / 2$ à 11 (p. 100) & 0 & 0 & 2,5 & 1,5 & 3,0 & 10,0 & 8,5 & 2,5 & 9,0 \\
\hline Plus de 11 (p. 100) & 1,0 & 1,5 & 0 & 0 & 0 & 3,5 & 0 & 0 & 3,0 \\
\hline \multirow{3}{*}{$\begin{array}{l}\text { Lait condensé et sec } \\
\text { Consommateurs (p. 100) } \\
\text { Consommateurs n'utilisant pas de lait } \\
\text { liquide (p. 100) }\end{array}$} & & & & & & & & & \\
\hline & 22,0 & 17,0 & 11,0 & 16,5 & 14,0 & 33,0 & 18,0 & 12,0 & 9,0 \\
\hline & 9,0 & 7,5 & 4,5 & 4,0 & 5,5 & 7,5 & 2,0 & 1,0 & 0 \\
\hline Total des consommateurs de lait (p. 100) & 60,5 & 60,0 & 67,5 & 64,5 & 85,5 & 91,0 & 83,0 & 88,5 & 97,0 \\
\hline Age (en années) & \multicolumn{2}{|c|}{$46-70$} & \multicolumn{2}{|c|}{$22-45$} & \multicolumn{2}{|c|}{$16-21$} & $12-15$ & $6-11$ & $3-5$ \\
\hline Sexe & Hommes & Femmes & Hommes & Femmes & Garçons & Filles & & & \\
\hline
\end{tabular}


lait mais aussi de produits laitiers frais, pour lesquels une fabrication familiale sera préférée à celle des yoghourts et des fromages blancs commerciaux.

b) En général, l'absence de consommation de lait est une décision d'ordre individuel. En effet, il y a un consommateur de lait dans 70 p. 100 des couples et au moins un consommateur dans 92 p. 100 des familles (tab. 5).

\section{TABLE 5}

Consommation de lait liquide en fonction de la taille du groupe familial

\begin{tabular}{l|c|c|c}
\hline & $\begin{array}{c}\text { Consommateurs } \\
\text { (p. 100) }\end{array}$ & $\begin{array}{c}\text { Non- } \\
\text { consommateurs } \\
\text { (p. 100) }\end{array}$ & $\begin{array}{c}\text { Présence de } \\
\text { consommateurs } \\
\text { dans }\end{array}$ \\
\cline { 2 - 4 } & 50,0 & 50,0 & \\
Célibataires & 58,0 & 42,0 & $70 \mathrm{p} .100$ des couples \\
Couples & 68,0 & 32,0 & $92 \mathrm{p} .100$ des familles \\
\hline Familles & & & \\
\hline
\end{tabular}

c) Dans la majorité des cas, les non-consommateurs de lait vivent dans un milieu familial où le lait est présent. Leur comportement relève donc d'un refus de consommation beaucoup plus que d'un manque de lait dans leur entourage immédiat.

d) La présence de consommateurs dans le groupe familial constitue une incitation à la consommation : chez les célibataires 50 p. 100 seulement des personnes utilisent du lait, dans les couples on en dénombre 58 p. 100 et dans les familles - où l'incitation est la plus forte - les consommateurs sont au nombre de 68 p. 100 (tab. 5).

Pourtant, même dans ce cas favorable, plus de 30 p. 100 des personnes ne font pas appel au lait pour leur alimentation.

Dans le domaine psychologique, il apparaît un réflexe de défense vis-à-vis des produits laitiers gras. Il s'intensifie avec l'âge, mais il est toujours nettement plus prononcé chez les femmes et même chez les jeunes filles que chez les hommes : il s'agit de la peur de prendre de l'embonpoint (cf. tab. 8). Il n'est pas possible de préciser si cette appréhension entraîne une réduction de la consommation des produits laitiers en général, ou si elle oriente la consommation vers les formes partiellement ou totalement dégraissées. Le seul fait précis 
est le choix du lait demi-écrémé, retenu par le $1 / 3$ des consommateurs ayant manifesté leur préférence et l'emploi de poudre de lait écrémé (tab. 4).

Le « goût » du lait est le principal motif invoqué pour justifier le refus de consommation (tab. 6). Ce défaut de qualité organoleptique vise le lait pasteurisé et plus encore celui qui a été stérilisé à haute température et dénommé couramment « de longue conservation $»(1)$.

La tendance à trouver un goût désagréable au lait pasteurisé est marauée au milieu de la vie, notamment chez les jeunes filles $(\mathrm{H})$ et les jeunes femmes (D). Elle disparaît presque après 45 ans. Une telle évolution semble indiquer une certaine accoutumance du consommateur au goût du lait pasteurisé. Mais il convient de rappeler que les personnes âgées sont celles qui s'abstiennent le plus aisément de consommer du lait.

En clair, ou bien on s'habitue, ou bien on renonce au lait pasteurisé !

La situation est différente en ce qui concerne le goût du lait « de longue conservation ». Ce produit, apparu beaucoup plus récemment sur le marché, suscite une opposition importante dans la plupart des catégories de consommateurs : à partir de 12 ans (J), les refus de consommation oscillent entre 14 et 35 p. 100 des individus (2).

Au total, le goût du lait est le principal obstacle à sa consommation. Ce motif est invoqué par 47 p. 100 des jeunes filles (H) et 44 p. 100 des jeunes femmes (D), les hommes des mêmes tranches d'âge paraissant moins sensibles à ce facteur (tab. 6).

(1) Le lait pasteurisé est chauffé à $63^{\circ} \mathrm{C}$ pendant $30 \mathrm{mn}$ ou à $72-80^{\circ} \mathrm{C}$ pendant $15 \mathrm{~s}$ en vue de détruire la flore pathogène. Le lait stérilisé est traité à des températures allant de $135^{\circ} \mathrm{C}$ à $145^{\circ} \mathrm{C}$ pendant des durées s'étalant entre 3 et $7 \mathrm{~s}$.

(2) Le nombre de refus concernant le lait de longue conservation, supérieur à celui visant le lait pasteurisé, peut surprendre en raison des intensités relatives des réactions chimiques au cours de la pasteurisation et de la stérilisation à haute température.

En théorie, un traitement conduit à haute température se justifie notamment par le fait qu'il entraîne des réactions chimiques moindres qu'une même opération effectuée à température plus basse.

Il en résulte que la stérilisation du lait à $140^{\circ} \mathrm{C}$ ne doit pas en altérer le goût dans des proportions notables. La modification organoleptique doit demeurer moindre que celle provoquée par une pasteurisation suivie d'une ébullition du lait par la ménagère, ce qui aboutit à un goût de " cuit " caractéristique.

Le consommateur accepte-t-il mieux ce dernier goût auquel il est habitué depuis des décennies et le considère-t-il même comme le goût "normal " du lait, ou bien le lait stérilisé subit-il une altération au cours de sa conservation, capable de diminuer sa qualité organoleptique? 


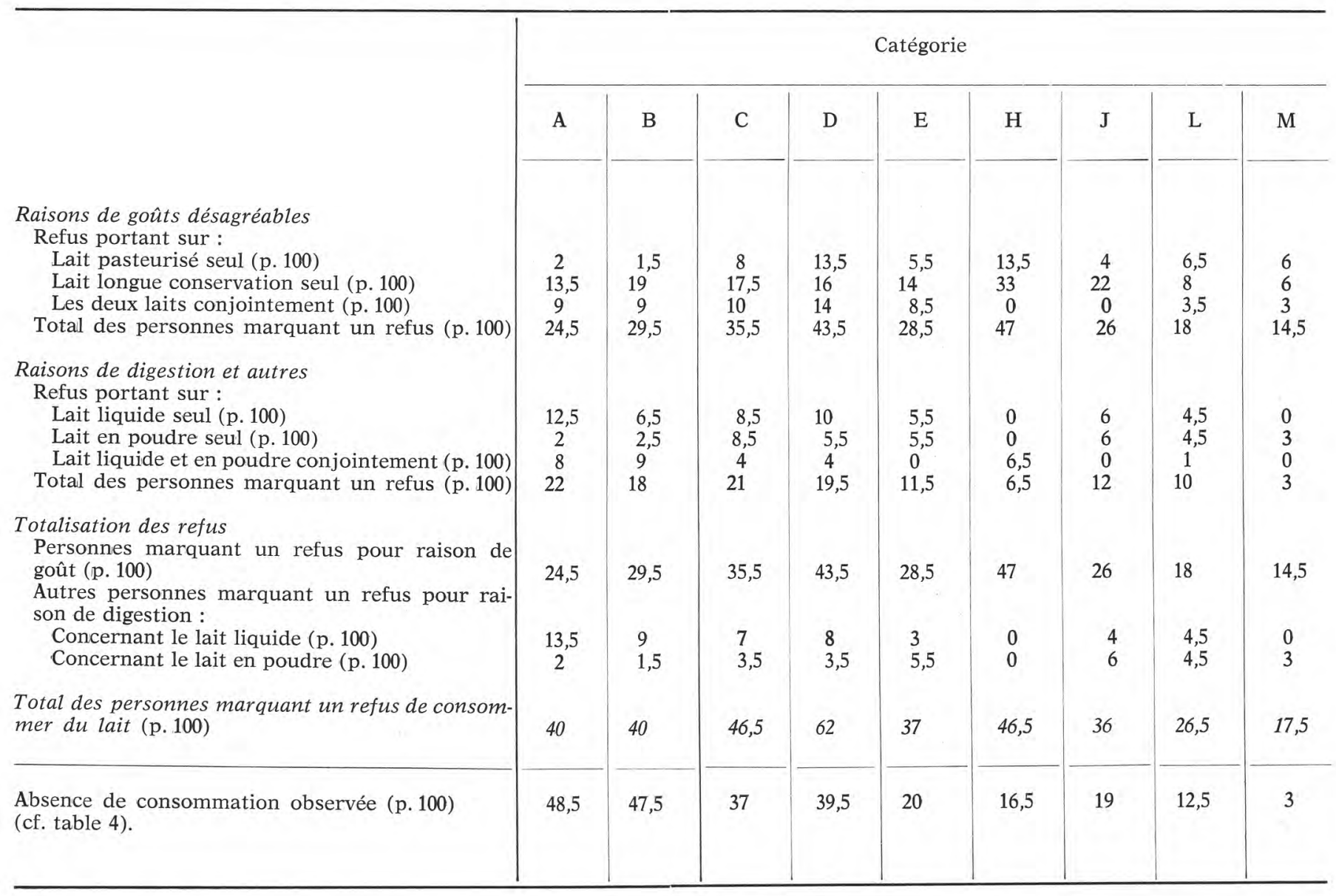


Comme ce motif ne croît pas avec l'âge, il ne peut expliquer la désaffection progressive vis-à-vis du lait.

Les refus pour "troubles digestifs " viennent en seconde position et se développent avec l'âge : ils sont invoqués par environ 20 p. 100 des adultes (A à D) et par 10 p. 100 des jeunes ( $\mathrm{E}$ à L) (tab. 6).

La responsabilité d'une déficience lactasique de l'intestin vient rapidement à l'esprit en raison de la croissance des troubles digestifs proportionnellement avec l'âge. Nous tenterons d'apporter une réponse à cette question.

Pour s'en tenir aux refus de consommation de lait, il est intéressant de comparer le nombre de motifs s'opposant à l'utilisation de ce produit et le nombre de personnes qui s'abstiennent effectivement de lait (tab. 6) : chez les adultes de plus de 45 ans (A et B), 40 p. 100 d'entre eux invoquent une raison de refuser le lait, mais les nonconsommateurs atteignent 48 p. 100 : il y a donc un petit pourcentage d'individus qui ne consomment pas de lait sans en avoir une raison bien définie.

Dans toutes les autres catégories (de C à M), la situation est inverse : le nombre de personnes qui ne consomment pas de lait demeure toujours nettement inférieur au nombre de motifs de refus. A titre d'exemple, 62 p. 100 des jeunes femmes (D) avancent un refus à la consommation de lait et pourtant 60,5 p. 100 d'entre elles en consomment effectivement. La situation est semblable chez les jeunes filles : 83,5 p. 100 de consommateurs malgré 46,5 p. 100 de motifs de refus. C'est dire qu'en dessous de 45 ans on s'oblige dans une certaine mesure à consommer du lait bien qu'on formule des raisons de le refuser. Au-delà de 45 ans, on se résigne beaucoup plus aisément à l'absence de lait, sans même en avancer obligatoirement un motif.

Cette situation découle, au moins partiellement de l'éducation et de l'image attachée au lait : il est nécessaire pour les enfants, pour la formation des os et des dents, etc. En un mot, c'est l'aliment de la croissance ou du jeune. Corollairement, plus on s'éloigne de cet état physiologique, plus les raisons de sa consommation s'estompent.

En conclusion, la diminution progressive de la consommation de lait au long de la vie provient de différentes causes dont les effets se cumulent :

- En premier lieu, le «goût » du lait est un obstacle sérieux à son acceptation; ce facteur est indépendant de l'âge.

- En second lieu, des troubles digestifs sont attribués à la consommation de lait; leur fréquence augmente avec l'âge.

- Enfin, le sentiment de sa nécessité sur le plan nutritionnel s'atténue très probablement au long de la vie. 


\section{CONSOMMATION DES PRODUITS LAITIERS FRAIS}

Sous cette rubrique figurent les yoghourts (laits fermentés) et les fromages blancs (caillé non-dégradé par l'affinage).

La consommation des yoghourts en fonction de l'âge se superpose tout à fait à celle du lait (cf. fig. 1) : entre 3 et 5 ans (M) on ne dénombre que 3 p. 100 de non-consommateurs; chez les hommes de plus de 45 ans cette valeur atteint 55 p. 100 (tab. 7).

Globalement sur 740 personnes, 65,4 p. 100 consomment des yoghourts. Ce produit est donc de grande consommation, sa diffusion égalant presque celle du lait. La diversité des produits actuellement offerts intervient sans aucun doute dans ce résultat.

La consommation des yoghourts présente cependant quelques légères différences avec celle du lait.

Ces produits sont sensiblement plus utilisés par les femmes que par les hommes : il y a 67 p. 100 de consommatrices et 56 p. 100 de consommateurs.

A l'inverse du lait également, il semble difficile de cerner les motifs de non-consommation. Les refus avoués sont en très petit nombre (tab. 8) : chez les adultes, environ 10 p. 100 de personnes invoquent une raison de "goût " ou de « digestion " pour refuser les yoghourts, les motifs étant légèrement plus importants chez les adultes de plus de 45 ans (A et B) que chez les plus jeunes (C et D). Les adolescents et les enfants n'expriment pratiquement pas de refus.

Les fromages frais représentent le produit laitier le moins fréquemment consommé par la population étudiée : les non-consommateurs vont de 48 p. 100 chez les tout-petits (M) à 72 p. 100 chez les hommes de plus de 45 ans (A) (tab. 7). Comme dans le cas du lait et du yoghourt, on assiste à une régression sensible en fonction de l'âge (cf. fig. 1).

Les femmes consomment plus volontiers les fromages frais (46 p. 100) que les hommes ( $36 \mathrm{p}, 100$ ). Mais la consommation des fromages blancs est assez irrégulière : il est presque exceptionnel que ces produits soient consommés de façon quotidienne.

Bien que les fromages frais ne soient pas un produit de très grande diffusion, ils ne suscitent pas de nombreux motifs de refus (tab. 8) : paradoxalement ce sont les produits laitiers les moins consommés et ceux pour lesquels le pourcentage de refus exprimés est le plus bas.

On peut conclure à une indifférence à leur égard, puisque sur 740 personnes, 442 ne consomment pas de fromages frais $(59,75$ p.100), sans qu'il y ait plus de 47 refus exprimés (6,35 p.100). 
TABLE 7

Consommation des yoghourts, des fromages frais et des fromages fermentés en fonction de la catégorie

Yoghourts

Pas de consommation (p. 100)

Un à trois par semaine (p. 100)

Un par jour (p. 100)

Deux par jour (p. 100)

Plus de deux par jour (p. 100)

\section{Fromages frais}

Pas de consommation (p. 100)

Une à trois fois par semaine (p. 100)

Une fois par jour (p. 100)

Deux fois par jour (p. 100)

\section{Fromages fermentés}

Pas de consommation (p. 100)

Une à trois fois par semaine (p. 100)

Une fois par jour (p. 100)

Deux fois par jour (p. 100)

\section{Catégorie}

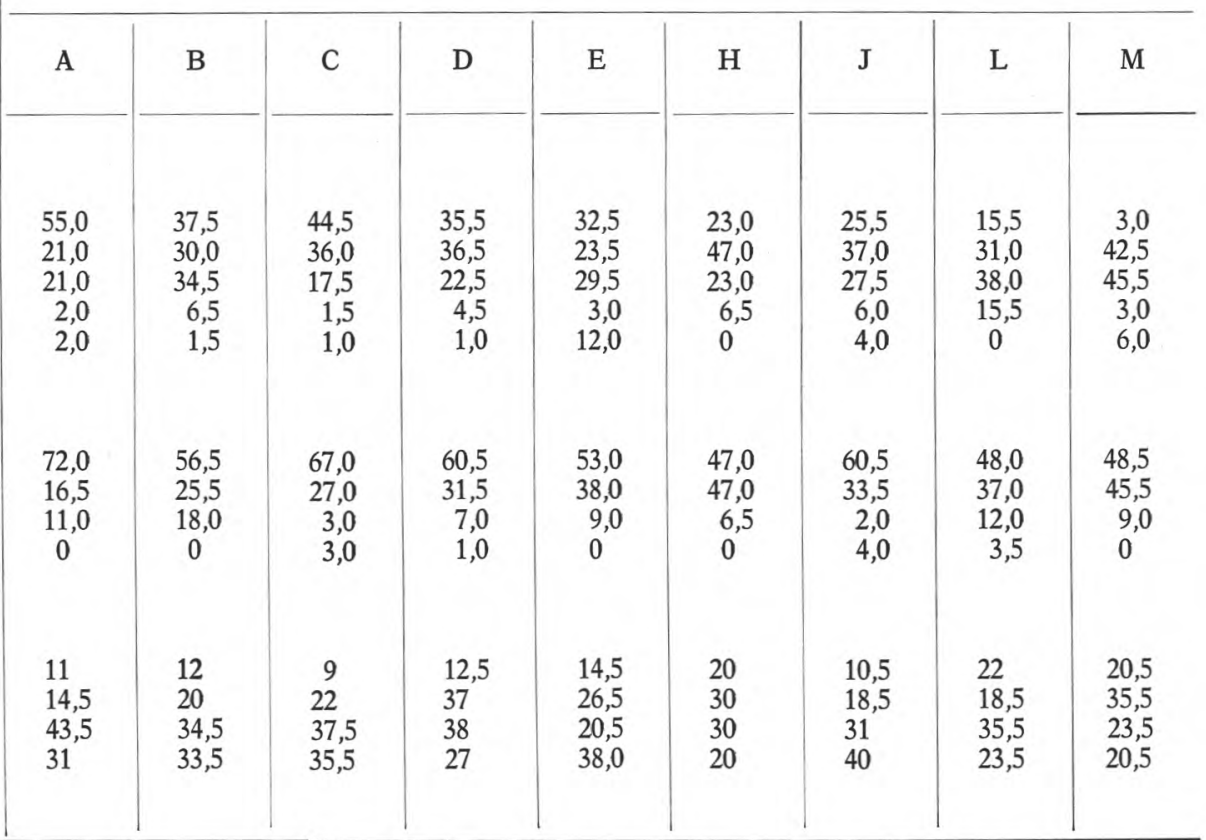


TABLE 7 (suite)

Consommation des yoghourts, des fromages frais et des fromages fermentés en fonction de la catégorie

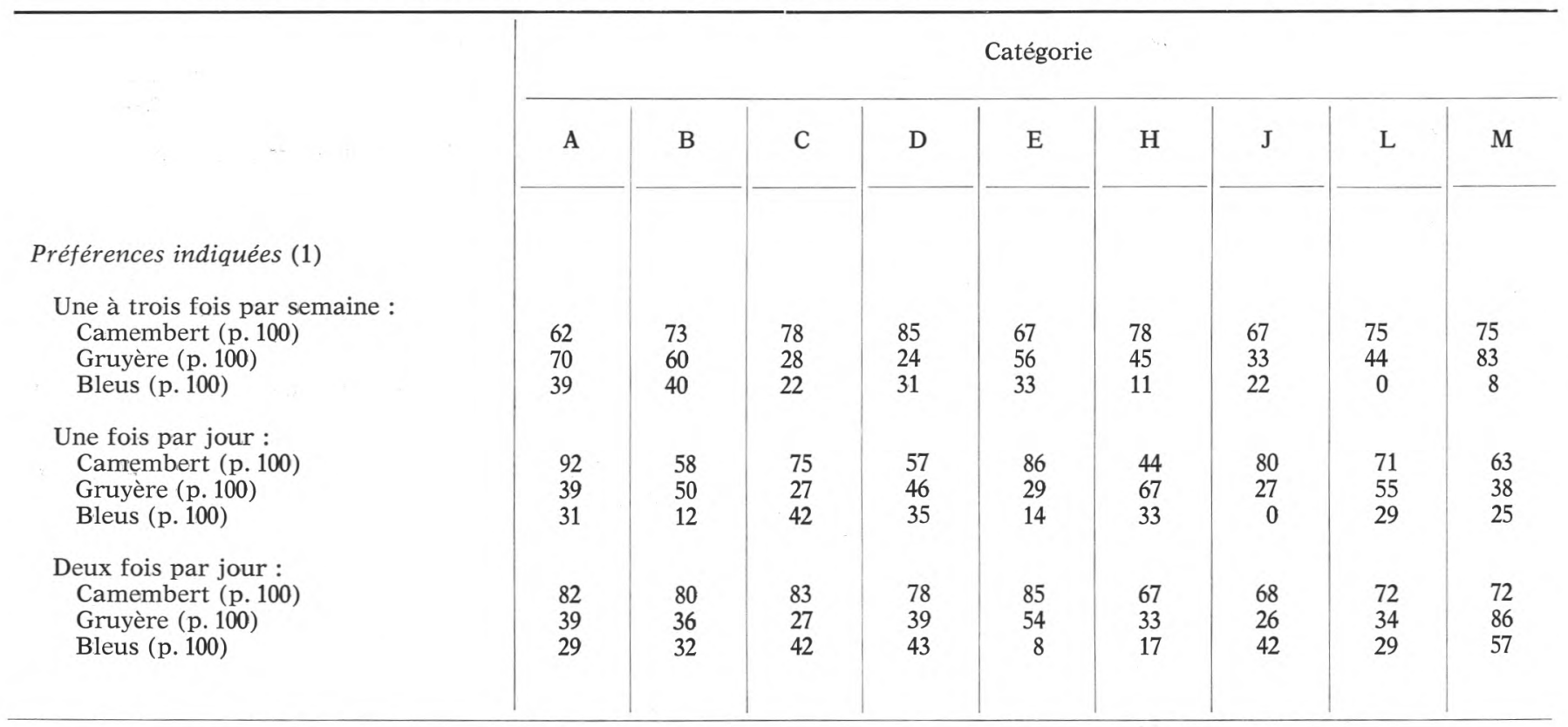

(1) Le total dépasse généralement 100 p. 100 , de nombreux consommateurs ayant cité plusieurs types de fromage. 
TABLE 8

Motifs s'opposant à la consommation de produits laitiers en fonction de la catégorie

\begin{tabular}{|c|c|c|c|c|c|c|c|c|c|}
\hline & \multicolumn{9}{|c|}{ Catégorie } \\
\hline & A & B & $\mathrm{c}$ & $\mathrm{D}$ & $\mathrm{E}$ & $\mathrm{H}$ & $\mathrm{J}$ & $\mathrm{L}$ & M \\
\hline Peur de grossir (p. 100) & 26,5 & 41 & 12 & 26,5 & 6 & 30 & 7 & 4,5 & 3 \\
\hline \multicolumn{10}{|c|}{ Raisons de goûts désagréables (p. 100) : } \\
\hline Yoghourts & 9 & 10 & 7 & 6 & 0 & 0 & 0 & 2 & 0 \\
\hline Fromages frais & 8 & 9 & 6 & 7,5 & 0 & 0 & 0 & 3 & 3 \\
\hline Fromages fermentés & 4,5 & 4 & 2 & 4 & 3 & 0 & 4 & 9 & 3 \\
\hline Total & 21,5 & 23 & 15 & 17,5 & 3 & 0 & 4 & 14 & 6 \\
\hline \multicolumn{10}{|c|}{ Raisons de digestion et autres (p. 100) : } \\
\hline Yoghourts & 4,5 & 2,5 & 2,5 & 1,5 & 0 & 3,5 & 2 & 0 & 0 \\
\hline Fromages frais & 3,5 & 2,5 & 1 & 3,5 & 0 & 3,5 & 0 & 0 & 0 \\
\hline Fromages fermentés & 5,5 & 2,5 & 0 & 5 & 0 & 3,5 & 0 & 1 & 0 \\
\hline Total & 13,5 & 7,5 & 3,5 & 10 & 0 & 10,5 & 2 & 1 & 0 \\
\hline
\end{tabular}




\section{CONSOMMATION DE FROMAGES FERMENTES}

Les fromages fermentés constituent le produit laitier le mieux accepté : 89 p. 100 des adultes et 80 p. 100 des jeunes en sont consommateurs. En tout, sur 730 personnes on ne compte que 97 non-consommateurs (13,3 p. 100) (tab. 7).

De plus, les fromages sont la seule forme de produit laitier dont la consommation tend à s'accroître avec l'âge (cf. fig. 1).

La fréquence de consommation est également remarquable : pour 70 p. 100 des adultes et pour 60 p. 100 des jeunes, les fromages entrent journellement dans l'alimentation. Même entre 3 et 5 ans, la moitié des enfants reçoit quotidiennement du fromage.

L'engouement pour ces produits est tel que dans les diverses catégories on dénombre de 20 à 40 p. 100 d'individus qui consomment des fromages deux fois par jour.

Il ne faut donc pas s'étonner que les motifs de refus soient rares (tab. 8) : dans aucune des catégories les oppositions exprimées dépassent 10 p. 100.

Un autre point mérite d'être souligné : la constance des préférences dans les diverses catégories de consommateurs (tab. 7) (3) : quels que soient l'âge, le sexe ou l'intensité de la consommation, les préférences vont toujours dans l'ordre suivant: Camembert $(75,5$ p. 100 des personnes), Gruyère (44,5 p. 100) et Bleus (26,5 p. 100). Seulement chez les jeunes enfants, on dénote une préférence plus marquée pour le Gruyère :

\begin{tabular}{|c|c|c|c|}
\hline & Hommes (A, C, E) & Femmes $(B, D, H)$ & Enfants (L, M) \\
\hline Camembert (p. 100) & 79 & 69 & 72 \\
\hline Gruyère (p. 100) & 41 & 45 & 57 \\
\hline Bleus (p. 100) & 29 & 28 & 25 \\
\hline
\end{tabular}

Traditionnellement, les fromages «forts » étaient consommés par les adultes, plutôt par les hommes, tandis que les fabrications

(3) Le total des préférences dépasse généralement 100 p. 100 étant donné que de nombreux consommateurs ont cité plusieurs types de fromages. 
plus « douces » étaient l'apanage des enfants. Les préférences pour le Gruyère rapportées ci-dessus en sont un reste.

La disparition de cette situation tient plus à une modification des caractéristiques organoleptiques des fromages qu'à une transformation du goût des consommateurs. Pour une très grande part, les Camemberts et les pâtes molles en général se présentent de plus en plus comme un produit doux et acceptable par tous, très éloigné des fabrications anciennes qui avaient beaucoup plus de saveur et de " corps ».

On peut probablement regretter que massivement les préférences se portent vers des fromages peu personnalisés au point de vue organoleptique; cette tendance constitue une incitation pour les producteurs à "banaliser " les qualités organoleptiques de leurs fromages.

Particulièrement dans le domaine de la consommation de fromages, les conclusions de notre enquête ne peuvent être extrapolées sans précautions à l'ensemble de la population française : la quasitotalité des personnes ayant répondu vit dans le Bassin parisien, région dans laquelle les fromages à pâte molle (Camembert, Brie) ont toujours bénéficié de la préférence des consommateurs.

Il est probable que les niveaux de consommation et la nature des préférences auraient pu différer sensiblement si l'enquête avait eu lieu dans d'autres régions.

Nous terminerons par une dernière remarque annexe à la consommation de fromages : ceux-ci étant consommés avec du pain, on pouvait se demander si le niveau de consommation de fromages pouvait influencer celui de la consommation de pain.

C'est pourquoi nous avons demandé la quantité de pain consommée (tab. 9). En rangeant les consommations de pain par rapport à celles de fromages, il n'en découle par une corrélation évidente :

\begin{tabular}{l|c|c}
\hline & $\begin{array}{c}\text { Non-consommateurs } \\
\text { de fromage }\end{array}$ & $\begin{array}{c}\text { Gros consommateurs } \\
\text { de fromages } \\
\text { (deux fois par jour) }\end{array}$ \\
\cline { 2 - 3 } & 55 & \\
Nombre de personnes & 24 & 151 \\
Consommation de pain (p. 100): & 33 & 4,5 \\
Pas ou très peu & 32 & 35 \\
Environ 125 g & 9 & 46 \\
Entre 125 et 250 g & 2 & 4 \\
Environ 250 g & & \\
Plus de 250 g & & \\
& & \\
\hline
\end{tabular}


TABLE 9. - Consommation de pain en fonction de la catégorie

Consommation de pain

Pas ou très peu (p. 100)

$125 \mathrm{~g} / \mathrm{j}$ (p. 100 )

125 à $250 \mathrm{~g} / \mathrm{j}$ (p. 100)

$250 \mathrm{~g} / \mathrm{j}($ p. 100$)$

Plus de $250 \mathrm{~g} / \mathrm{j}$ (p. 100)

Pain consommé (1)

Consommation de $125 \mathrm{~g}$ :

Pain blanc (p. 100)

Pain complet (p. 100)

Biscottes (p. 100)

Consommation de 125 à $250 \mathrm{~g}$ :

Pain blanc (p. 100)

Pain complet (p. 100)

Biscottes (p. 100)

Consommation de $250 \mathrm{~g}$ :

Pain blanc (p. 100)

Pain complet (p. 100)

Biscottes (p. 100)

\begin{tabular}{|c|c|c|c|c|c|c|c|c|}
\hline A & B & C & D & E & $\mathrm{H}$ & $\mathrm{J}$ & $\mathrm{L}$ & M \\
\hline $\begin{array}{c}7 \\
43 \\
27 \\
18 \\
5,5\end{array}$ & $\begin{array}{l}31 \\
45 \\
15,5 \\
7 \\
1,5\end{array}$ & $\begin{array}{l}10 \\
33 \\
34 \\
14,5 \\
8,5\end{array}$ & $\begin{array}{r}15,5 \\
59 \\
19,5 \\
4,5 \\
1,5\end{array}$ & $\begin{array}{c}6 \\
17,5 \\
23,5 \\
29,5 \\
23,5\end{array}$ & $\begin{array}{c}20,5 \\
48,5 \\
17 \\
3,5 \\
10,5\end{array}$ & $\begin{array}{c}10,5 \\
34 \\
36 \\
6,5 \\
13\end{array}$ & $\begin{array}{l}17,5 \\
53 \\
23 \\
6 \\
1\end{array}$ & $\begin{array}{c}30,5 \\
69,5 \\
0 \\
0 \\
0\end{array}$ \\
\hline $\begin{array}{r}74 \\
8 \\
16\end{array}$ & $\begin{array}{r}69 \\
6 \\
38\end{array}$ & $\begin{array}{r}74 \\
16 \\
6\end{array}$ & $\begin{array}{r}73 \\
9 \\
19\end{array}$ & $\begin{array}{r}83 \\
0 \\
0\end{array}$ & $\begin{array}{l}86 \\
14 \\
14\end{array}$ & $\begin{array}{r}81 \\
12 \\
6\end{array}$ & $\begin{array}{r}87 \\
2 \\
11\end{array}$ & $\begin{array}{r}91 \\
9 \\
17\end{array}$ \\
\hline $\begin{array}{r}83 \\
21 \\
0\end{array}$ & $\begin{array}{r}81 \\
18 \\
0\end{array}$ & $\begin{array}{r}90 \\
6 \\
14\end{array}$ & $\begin{array}{l}85 \\
15 \\
12\end{array}$ & $\begin{array}{r}62 \\
12 \\
0\end{array}$ & $\begin{array}{r}100 \\
0 \\
0\end{array}$ & $\begin{array}{r}77 \\
0 \\
0\end{array}$ & $\begin{array}{r}75 \\
20 \\
0\end{array}$ & \\
\hline $\begin{array}{r}75 \\
6 \\
6\end{array}$ & $\begin{array}{r}100 \\
20 \\
20\end{array}$ & $\begin{array}{r}86 \\
14 \\
5\end{array}$ & $\begin{array}{r}100 \\
12 \\
0\end{array}$ & $\begin{array}{r}100 \\
13 \\
25\end{array}$ & & & & \\
\hline
\end{tabular}

(1) Le total peut dépasser $100 \mathrm{p} .100$, des personnes ayant indiqué plusieurs formes de produit céréalier. 


\section{TABLE 10}

Consommation des dérivés laitiers chez les adultes en fonction de la consommation de lait

\begin{tabular}{|c|c|c|c|c|c|c|c|c|}
\hline \multirow[b]{2}{*}{ Niveau de consommation du lait } & \multicolumn{3}{|c|}{ Hommes ( $\mathrm{A}$ et $\mathrm{C}$ ) } & \multicolumn{3}{|c|}{ Femmes (B et D) } & \multicolumn{2}{|c|}{$\begin{array}{l}\text { Consommation } \\
\text { pour l'ensemble des } \\
\text { catégories }\end{array}$} \\
\hline & nul & $<250 \mathrm{ml}$ & $>250 \mathrm{ml}$ & nul & $<250 \mathrm{ml}$ & $>250 \mathrm{ml}$ & $\mathrm{A}$ et $\mathrm{C}$ & B et $\mathrm{D}$ \\
\hline \multicolumn{9}{|c|}{ Pourcentage de consommateurs de : } \\
\hline \multicolumn{9}{|l|}{ Yoghourts } \\
\hline Total des consommateurs & 41 & 51 & 68 & 58 & 80 & 82 & 50 & 64 \\
\hline Un yoghourt par jour & 16 & 16 & 30 & 19 & 25 & 24 & 19 & 24 \\
\hline \multicolumn{9}{|l|}{ Fromages fermentés } \\
\hline Total des consommateurs & 93 & 87 & 85 & 91 & 85 & 97 & 90 & 88 \\
\hline Une à trois fois par semaine & 17 & 22 & 22 & 18 & 22 & 13 & 18 & 29 \\
\hline Deux fois par jour & 33 & 33 & 17 & 30 & 29 & 23 & 33 & 30 \\
\hline
\end{tabular}


Une consommation élevée de fromages se révèle incompatible avec une absence de consommation de pain. Mais, à part cette observation, l'engouement pour le fromage ne semble pas influencer le taux de consommation de pain.

\section{CONSOMMATION DE PRODUITS LAITIERS ET INSUFFISANCES LACTASIQUES}

Les consommateurs de lait sont-ils, ou non, également des amateurs de yoghourts et de fromages ? Pour répondre à cette question, nous avons classé dans la table 10 les fréquences de consommation de dérivés laitiers en fonction du niveau de consommation de lait.

A première vue, les non-consommateurs de lait ne comblent pas cette absence alimentaire par une consommation plus forte des autres produits laitiers : les consommations de yoghourts et de fromages ne paraissent pas étroitement liées à celle du lait.

Cependant quelques tendances se font jour :

- La consommation de yoghourts paraît proportionnelle à celle du lait : les grands buveurs de lait consomment plus volontiers des yoghourts et en quantité plus importante que les non-consommateurs de lait.

Le parallélisme entre la consommation de ces deux produits sera illustré dans la figure 1.

- Les relations entre la consommation de lait et celle de fromages fermentés sont encore plus ténues : il y aurait une très faible tendance à enregistrer un antagonisme entre ces deux produits en ce sens que les grands consommateurs de lait consomment moins volontiers des fromages à chaque repas (deux fois par jour).

En utilisant d'autres critères, la figure 1 montre une évolution inverse de la consommation de lait et de fromages.

Il est intéressant d'examiner plus particulièrement la situation des personnes refusant le lait (tab. 11) ; rappelons qu'elles constituent plus de 30 p. 100 de la population enquêtée.

Chez les jeunes ne consommant pas de lait, les autres produits laitiers suppléent facilement l'absence de lait : plus des 3/4 de ces jeunes utilisent des yoghourts et des fromages fermentés.

Chez les adultes, les substitutions se font moins aisément et, dans ce cas, le seul dérivé laitier couramment accepté est le fromage. Les consommations de produits laitiers frais demeurent faibles.

$\mathrm{Au}$ total, on aboutit à une absence de produits lactosés (lait et yoghourt) chez 45 adultes âgés (A et B), 50 adultes plus jeunes $(C$ et $D)$ et 24 jeunes ( $E$ à $M)$. Ces effectifs représentent 27 p. 100, 15 p. 100 et 10 p. 100 des catégories concernées. 
TABLE 11

Consommation de produits laitiers chez les non-consommateurs de lait

\begin{tabular}{|c|c|c|c|c|c|}
\hline & \multicolumn{2}{|c|}{ Adultes $46-70$ ans } & \multicolumn{2}{|c|}{ Adultes $22-45$ ans } & \multirow{2}{*}{$\begin{array}{c}\text { Jeunes } \\
(21-3 \text { ans } \\
\text { (E à } M)\end{array}$} \\
\hline & $\underset{\text { (A) }}{\text { Hommes }}$ & $\underset{(B)}{\text { Femmes }}$ & $\underset{\text { (C) }}{\text { Hommes }}$ & $\underset{\text { (D) }}{\text { Femmes }}$ & \\
\hline Nombre de personnes ne consommant pas de lait & 43 & 36 & 56 & 71 & 33 \\
\hline $\begin{array}{l}\text { Consommateurs de: } \\
\quad \text { Yoghourts (p. 100) } \\
\text { Fromages frais (p. 100) } \\
\text { Fromages fermentés (p. 100) }\end{array}$ & $\begin{array}{l}39 \\
28 \\
91\end{array}$ & $\begin{array}{l}47 \\
39 \\
92\end{array}$ & $\begin{array}{l}43 \\
50 \\
96\end{array}$ & $\begin{array}{l}75 \\
56 \\
94\end{array}$ & $\begin{array}{l}76 \\
48 \\
88\end{array}$ \\
\hline $\begin{array}{l}\text { Personnes ne consommant: } \\
\text { Ni lait, ni yoghourts, ni fromages frais (p. 100) } \\
\text { Ni lait, ni yoghourts, ni fromages frais, ni } \\
\text { fromages fermentés (p. 100) }\end{array}$ & $\begin{array}{r}47 \\
5\end{array}$ & $\begin{array}{r}42 \\
0\end{array}$ & $\begin{array}{r}29 \\
0\end{array}$ & $\begin{array}{r}11 \\
0\end{array}$ & $\begin{array}{r}15 \\
9\end{array}$ \\
\hline Pas de produits lactés (lait et yoghourt) (p. 100) & 61 & 53 & 57 & 25 & 24 \\
\hline $\begin{array}{l}\text { Nombre de personnes ne consommant pas de } \\
\text { produits lactosés }\end{array}$ & 26 & 19 & 32 & 18 & 8 \\
\hline
\end{tabular}


Il serait aventureux de prétendre qu'un défaut de lactase intestinale - consciemment ressenti ou non - soit le seul responsable de ce comportement alimentaire. A l'inverse, ces pourcentages constituent indiscutablement les fractions maximales de la population dans lesquelles une telle insuffisance enzymatique pourrait éventuellement être décelée.

Si on souhaite avancer une estimation plus précise des déficiences lactasiques, nous disposons de deux sources d'information :

- Sur 501 adultes des catégories A à D, 52 ont dans leur ascendance une personne connue pour avoir refusé le lait. Parmi ces 52 personnes, 13 d'entre elles ne consomment pas de lait.

En admettant que ces refus dans des générations successives soient imputables au caractère transmissible de la déficience en lactase, il y aurait 2,6 p. 100 des adultes enquêtés qui souffriraient de cette affection.

- En totalisant les refus de consommation pour " raisons de digestion » (tab. 6), portant spécifiquement sur les différentes formes de lait, c'est-à-dire en comptabilisant les personnes refusant le lait et acceptant les fromages (produits sans lactose) et les yoghourts (produits à taux de lactose réduit et renfermant une lactase), on aboutit aux conclusions suivantes : 19 adultes sur $501(3,8 \mathrm{p} .100)$ et 1 jeune $(0,4 \mathrm{p} .100)$ pourraient souffrir d'une intolérance au lactose, telle que l'individu l'ait empiriquement décelée et l'ait combattue en éliminant les laits de son alimentation. Parmi ces 19 adultes, l'un d'entre eux précise qu'effectivement ses troubles digestifs sont imputables au lactose.

En conclusion, les estimations établies à partir de cette enquête confirment que l'intolérance au lactose croît avec l'âge.

De plus, parmi les adultes enquêtés - au plus - 20 p. 100 pourraient présenter une éventuelle insuffisance lactasique et seulement 3 à 4 p. 100 souffriraient d'une déficience lactasique marquée.

Selon cette enquête, les intolérances au lactose semblent donc demeurer assez rares, même chez les adultes; elles ne peuvent être tenues pour responsables d'une absence de consommation de lait chez 30 p. 100 des individus.

Mais, encore une fois, ce ne sont-là que des estimations obtenues par voie indirecte à partir d'un questionnaire. Elles ne sauraient être prises comme une conclusion définitive et absolue.

\section{CAS DES PERSONNES AGEES}

En raison du faible nombre de réponses concernant les personnes âgées (plus de 70 ans), les résultats obtenus ne peuvent être totalement significatifs, d'autant plus que ces personnes vivent dans 
un milieu familial, situation peut-être privilégiée par rapport à nombre de personnes du même âge.

Les réponses obtenues constituent donc une information que nous résumerons brièvement.

Les personnes âgées consomment peu de lait : aucune ne dépasse $1 / 4$ de litre par jour. Les non-consommateurs sont au nombre de 40 p. 100 et les personnes utilisant moins de $1 / 4$ de litre représentent également $40 \mathrm{p} .100$. Trois personnes sur 17 ne reçoivent aucune forme de lait.

Les refus de consommation sont très mal exprimés : peut-être parce que les questionnaires ont été remplis par des personnes connaissant mal l'opinion de cette catégorie. Peut-être également parce que - comme les adultes de plus de 45 ans - les gens âgés refusent le lait sans motivations très précises. Il est probable que le comportement de ces personnes ne fait que prolonger celui des adultes de plus de 45 ans (A et B).

En ce qui concerne les produits laitiers frais, la consommation est également faible : seulement 40 p. 100 consomment des yoghourts et 35 p. 100 des fromages blancs.

Les fromages fermentés sont la seule forme utilisée couramment: il y a 82 p. 100 de consommateurs. De plus 75 p. 100 des personnes âgées consomment quotidiennement du fromage et 30 p. 100 d'entre elles en consomment même deux fois par jour.

Le Camembert ( 59 p. 100 des choix) et le Gruyère (53 p. 100) se partagent les préférences; les mentions en faveur des fromages Bleus ne constituent que 20 p. 100 des opinions exprimées.

Au total, selon ces indications, le comportement des personnes âgées ne se distingue pas nettement de celui des adultes de 45 à 70 ans (A et B) : la consommation en lait et produits laitiers frais demeure faible, celle des fromages est la seule à être importante.

Nous voulons, pour terminer, mentionner les habitudes alimentaires de la doyenne de cette enquête, âgée de 107 ans : elle consomme peu de lait (moins de 1/4 de litre), mais environ deux yoghourts par jour, du fromage frais (environ une fois par jour) et du fromage fermenté (deux fois par jour). Ces produits laitiers, d'une consommation et d'une digestion aisées, lui fournissent à eux seuls la majeure partie des protéines, vitamines et minéraux dont elle a besoin. Un simple complément en aliments énergétiques suffit pour atteindre un équilibre alimentaire satisfaisant.

\section{Conclusions et résumé}

L'enquête porte sur une population se rattachant aux " classes moyennes ". Les résultats globaux portent sur 239 jeunes (entre 


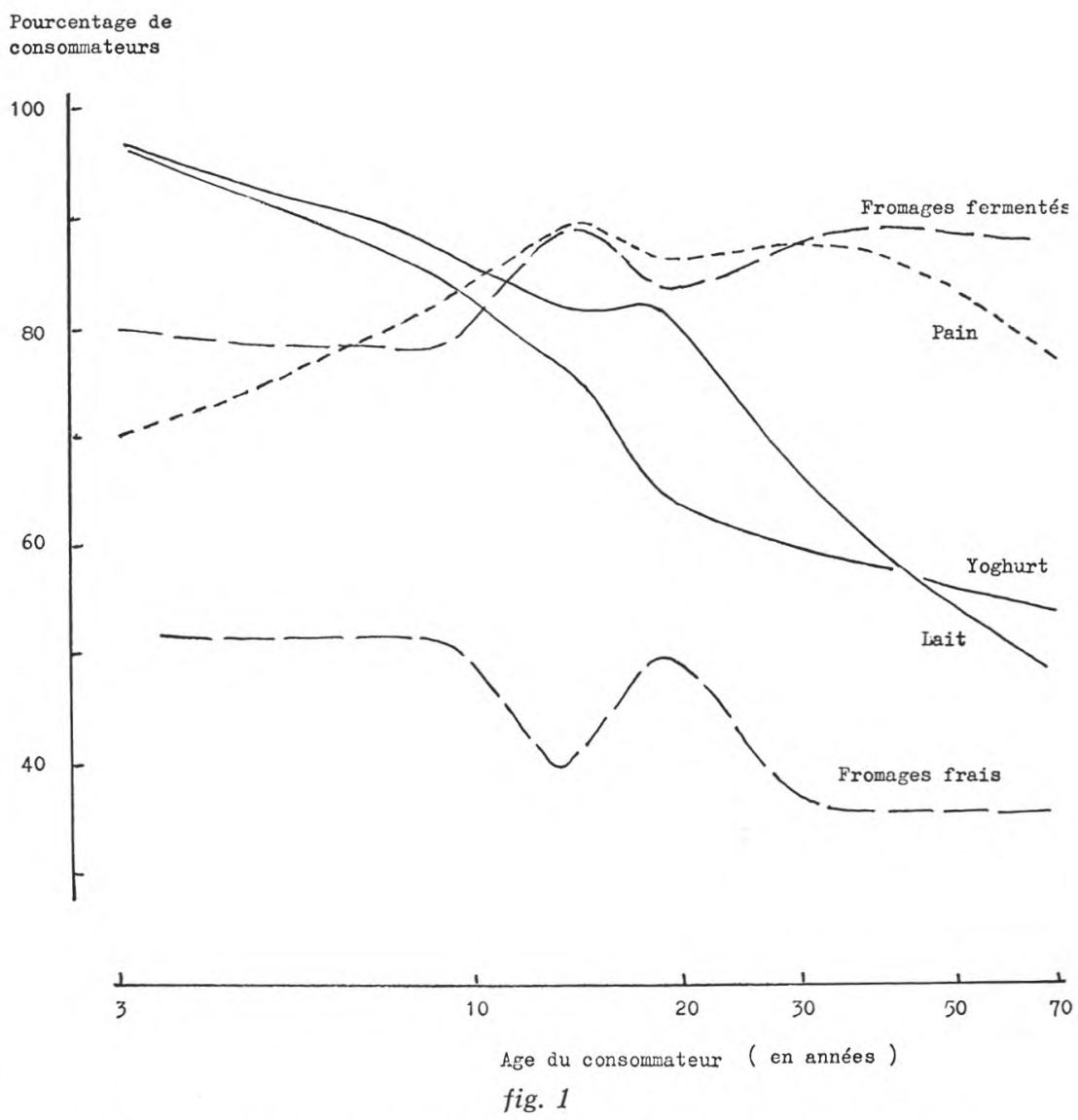

Consommation des produits laitiers et du pain en fonction de l'âge.

3 et 21 ans), 501 adultes (de 22 à 70 ans) et 17 personnes de 71 ans et plus.

Sur ces 757 personnes, 40 p. 100 seulement consomment des fromages frais, 64,5 p. $100 \mathrm{du}$ lait liquide, 65 p. $100 \mathrm{du}$ yoghourt et 69,5 p. 100 une des formes commerciales de lait. Les fromages fermentés constituent le seul produit laitier de très grande diffusion, acceptés par 87 p. 100 des personnes ; leur consommation est souvent quotidienne.

La consommation des produits laitiers varie grandement en fonction de l'âge, comme le rapporte la figure 1 .

La consommation de lait et de yoghourt baisse régulièrement et solidairement au cours de la vie. Si les consommations de fromages 
sont plus stables, celle de fromages blancs tend à diminuer et celle des fromages fermentés à s'accroître. Ces phénomènes apparaissent dans les corrélations ci-dessous :

Consommateurs de lait - Consommateurs de yoghourts

Consommateurs fromages frais - Consommateurs fromages fermentés

Age du consommateur - Consommateurs de lait

Age du consommateur - Consommateurs de yoghourts

Age du consommateur - Consommateurs de fromages frais

Age du consommateur - Consommateurs de fromages fermentés

Degré

de

corrélation

$-0,84$

$-0,97$

$-0,96$

$-0,74$

$+0,62$

Par ailleurs, la représentation graphique de ces consommations en fonction de l'âge fait apparaître un point de rupture dans toutes les courbes vers la fin de l'adolescence, entre 15 et 20 ans. Seule la consommation de pain semble échapper à ce phénomène.

Si une telle évolution est significative, elle traduit l'importance biologique de la "crise de croissance » qui provoque en réalité un bouleversement profond de l'individu, allant jusqu'à une perturbation temporaire de ses préférences et de ses habitudes alimentaires.

Le fromage fermenté est à la fois le produit le plus consommé et celui pour lequel on manifeste de moins en moins de motifs de refus ; à l'inverse le fromage frais est le moins consommé sans pour autant faire l'objet d'un nombre appréciable de motifs de refus.

La consommation du lait est la seule à se trouver face à une opposition très clairement exprimée et se décomposant de la façon suivante : le principal motif invoqué est le "goût » du lait (surtout dans le cas du lait de longue conservation). Le second type de refus a trait aux troubles digestifs attribués à la consommation de lait, cette motivation se développant avec l'âge.

Indiscutablement, on assiste à une désaffection vis-à-vis du lait, progressive tout au long de la vie.

Quelques nuances distinguent le comportement des hommes et des femmes. Si la consommation effective du lait est indépendante du sexe, les femmes le consomment avec plus de réticence, car « la peur de grossir » et « les refus pour raisons de goût » sont plus souvent invoqués par elles que par les hommes.

Elles consomment plus volontiers les produits laitiers frais, yoghourt et fromages frais. Par contre, on n'enregistre aucune différence entre les sexes en ce qui concerne les fromages fermentés: 
dans tous les cas les fromages à pâte molle (Camembert) viennent en tête des préférences, suivies d'assez loin par les fromages à pâte cuite (Gruyère) ; les fromages persillés (Bleus) n'entrent que pour une faible part dans les préférences de cette enquête portant essentiellement sur une population vivant dans la région parisienne.

En considérant les refus de consommation des produits lactosés, soit spontanés, soit appuyés sur des troubles digestifs, il est possible d'avancer prudemment des estimations relatives à l'existence de défauts de lactase intestinale. Ceux-ci sont presque inexistants chez les jeunes et se développent avec l'âge chez les adultes.

On peut avancer que 3 à 4 p. 100 des adultes sont susceptibles de souffrir d'une déficience lactasique importante. Des insuffisances légères ne semblent pas pouvoir être décelables dans plus de 20 p. 100 des adultes, estimation maximale probablement nettement supérieure à la réalité.

Ces défauts d'équipement enzymatique ne peuvent expliquer à eux seuls la diminution de la consommation de lait en fonction de l'âge.

\section{S u $\mathbf{m} \mathbf{m}$ a r y}

The inquiry is about " middle class " people. The results concern 239 young people (between 3 and 21 years old), 501 adults (between 22 and 70) and 17 persons older than 70.

Among these 757 persons, only 40 p. 100 consume cottage cheese, 64.5 p. 100 liquid milk, 65 p. 100 yoghurt and 69.5 p. 100 one of the commercial forms of milk. Only the fermented cheese is widely diffused and accepted by 87 p. 100 of persons. It is often eaten every day.

The consumption of dairy products differs according to the age as it is shown in the fig. 1. The milk and yoghurt consumption regularly drops thoroughly life. If the cheese consumption is more constant, this of cottage cheese decrease and this of fermented cheese increase.

These facts appear in the following correlations:

Milk consumers - Yoghurt consumers

Cottage cheese consumers - Fermented cheese consumers

Degree of correlation

(r)

Consumer's age - Milk consumers

Consumer's age - Yoghurt consumers

Consumer's age - Cottage cheese consumers

Consumer's age - Fermented cheese consumers 
Besides, the graphic representation of these consumptions according to the age makes appear a rupture point in all the curves, approximately in the end of the adolescence, between 15 and 20 . Only the bread consumption seems to be an exception to this phenomenon.

If such an evolution is significant, it shows the biological importance of the "growth crisis " that really provokes a deep disorder. This crisis can involve a temporary perturbation in the adolescent's preferences and food customs.

The fermented cheese is both the most consumed product and that is the last refused. On the contrary, the cottage cheese is the least consumed, without expressed reasons.

The milk consumption is the only one to be found in front of an opposition clearly expressed, decomposed as follows: the main reason is the "taste " of milk (especially in the case of U.H.T. milk). The second type of refusal refers to digestive disorders attributed to the milk consumption, this motive increases with the age.

Indisputably, there is a disaffection towards the milk, progressive during the life.

For some reasons, women and men behave differently. Though the milk consumption does not depend on sexes, women are more reticent to consumr it because they are afraid to put on weight and they often do not appreciate its " taste ".

They prefer fresh dairy products (yoghurt and cottage cheese).

On the other hand, men as women eat fermented cheese: in all cases, soft cheese (Camembert) are the most preferred, then come further baked-paste cheese (Swiss cheese). Blue-moulded cheese is the less one appreciated. But, this inquiry mainly concerns the Parisian district.

Considering therefusal of lactose-containing dairy products consumption, spontaneous or based on digestive disorders, we may estimate the lack of intestinal lactase: it is especially no-existing in the young people and developping little by little when people become older.

We can think that 3 to 4 p. 100 of adults can suffer from important lactase deficiency. Slight insufficiencies cannot be revealed in more than $20 \mathrm{p} .100$ of adults, maximum estimation probably very superior to the reality.

These lacks of enzymic equipment cannot explain the diminution of the milk consumption according to the age.

\section{Remerciements}

Sur le plan budgétaire, ce travail est exemplaire car les dépenses qu'í: a occasionnées se montent aux fournitures nécessitées par la constitution des questionnaires : 1800 feuilles ronéotypées et 600 agrafes. 
Par contre, il a demandé un effort aux 274 personnes qui ont pris la peine, et le temps, de remplir les questionnaires. Je souhaite que les résultats obtenus justifient, a posteriori, leur bonne volonté.

Toute cette enquête a reposé, en fait, sur la collaboration amicale des intermédiaires qui ont accepté de jouer le rôle ingrat de sergent recruteur en suscitant la participation à l'enquête et en se chargeant de la diffusion et du ramassage des questionnaires. Que M. et Mme Frangne, Mlle Tourneur, MM. Claveau, Créac'h, Drapron, Marcellin, ainsi que M. Lorrain et le Comité d'Entreprise de Rhône-Poulenc en soient vivement remerciés.

Enfin, pour le dépouillement, j'ai apprécié l'aide efficace de Catherine Adrian, à qui vont également mes remerciements.

Reçu pour publication en janvier 1977.

\section{Références}

De nombreuses enquêtes et statistiques ont eu pour objet les produits laitiers ; elles sont relatives aux taux de consommation ou aux motivations du consommateur à leur égard.

Nous pensons utile d'en citer les principales pour la période 1955-1975.

O.C.D.E. (1975). - Statistiques de la consommation des denrées alimentaires (1955-1973), Paris.

Q.C.D.E. (1975). - Bilans du lait et des produits laitiers dans les pays membres de l'O.C.D.E., Paris.

Cook (J.), Altman (D. G.), Jacoby (A.), Holland (W. W.) and Elliot (A.) (1975). The contribution made by school milk to the nutrition of primary schoolchildren. Brit. J. nutrit., 34, 91-103.

MARIANI (A.) (1972). - Consumption of milk in Italy. Latte, 46, 478-482.

Raunikar (R.) and Purcell (J. C.) (1972). - Trends in the milk market. Res. rep., College Agric. exp. Stat., Univ. Georgia, n ${ }^{\circ} 139,26$ p.

Consumption statistics for milk and milk products (1968-1969). Ann. Bull. Intern. Dairy Fed., 1971, n 5, 95 p.

Van SchaIK (T. F. S. M.) and De Roon (D. E.) (1971). - Diet Study in 2000 households in the Netherlands. 7. Individual intakes of cheese. Voeding, 32, 403-405.

Van Schaik (T.F.S. M.) and De Roon (D. E.) (1971). - Consumption of butter, different margarines, halvarine and other fats. Voeding, 32, 433-438.

Wigbout (M.) (1971). - Diet study in 2000 households in the Netherlands. 8. Average consumption of milk and yoghurt according to a number of criteria. Voeding, 32, 555-568.

Claudian (J.) et Serville (Y.) (1970). - Réflexions sur la consommation des produits laitiers en France. Bull. INSERM, 25, 213-258.

Consumption statistics for milk and milk products (1966-1967). Ann. Bull. Intern. Dairy Fed., 1969, Pt 5, 109 p.

O.C.D.E. (1968). - Perspectives du marché des produits laitiers. Agr., 3, Paris.

Pequignot (G.) et Pequignot (E.) (1969). - Enquête sur l'acceptation du laitboisson à l'hôpital. Bull. INSERM, 24, 1579-1596.

Gruebele (J.W.) (1968). - Milk consumption in Illinois schools (1954-1966). Univ. Illinois agric. exp. Stat. Bull., $\mathrm{n}^{\circ} 732$, june, $27 \mathrm{p}$.

Purcell (J. C.), Raunikar (R.) and Elrod (J.C.) (1968). - Analysis of demand for beverage milk. Univ. Georgia College agric. exp. Stat. res. Bull., $\mathrm{n}^{\circ} 43$, oct., $28 \mathrm{p}$. 
CiPRA (J. E.) and Tinklin (G. L.) (1969). - Milk consumption in a Kansas « class A " city. J. home econ., 61, 49.

Mc Kensie (J. C.), Mattinson (J.) and YudKin (J.) (1967), - Milk in schools : an experiment in nutrition education. Brit. J. nutrit, 21, 811-817.

Trémolières (F.), Claudian (J.) et Serville(Y.) (1966), - Consommation des fromages. Cahiers nutrit. diét., 1, 47-49.

Trémoliéres (F.), Claudian (J.) et Serville (Y.) (1965), - Consommation des fromages à Marseille. Bull. INSERM, 20, 675-733.

AverbaCH (J.) et al. (1964). - Studies on the milk consumed in the federal capital. Rev. Assoc. med. argentina, 78, 257-270.

Baines (A. H. J.), Hollingsworth (D. F.) and Leitch (I.) (1963). - Diets of working-class famillies with children before and after the second word war, Nutrit. Abstr. Rev., 33, 653-668.

De WIJN (J.F.) (1962), - Health aspects of milk consumption in relation to the changing consumption pattern in the Netherlands. Netherlands melk zuivel tijdschr., 16, 226-251.

Wrrths (W.) (1961). - Consumption of milk in the Federal Republic. Med. Ernährung, 2, 143.

White (J.V.) (1959). - Consumption of dairy products in New Zealand. N.Z. J. agric., 99, 525-530.

15 Congr. intern. Lait., Londres (1959). - Niveaux de consommation du lait et des produits laitiers. 4, sect. 6, 2313-2316.

Coles (J. V.) (1959). - Consumption of dairy products by urban families in California. Calif. agric. exp. Stat. Bull., $\mathrm{n}^{\circ} 767$, april, $141 \mathrm{p}$.

EMPSON (J.D.) (1958). - Economics, market research and the market for milk. J. agric. écon., 13, 169-182.

RoBeRTs (J. B.) (1958). - Consumption of milk and dairy products in Kentucky markets. Kentucky agric. exp. Stat. Bull., $\mathrm{n}^{\circ} 660$, june, $31 \mathrm{p}$.

EMPSON (J.D.) (1958). - The variation in the per capita consumption of milk between towns in Great Britain, and their causes. J. dairy res., 25, 397-406.

EMPSON (J.D.) (1957). - The utilization of milk in the home. Farm. economist, 8, $\mathrm{n}^{\circ} 11-12,15-30$.

Newman (H.H.) (1957), - The milk and water intake of small children. Arch. pediatr., 74, 456-462.

Mirone (L.) and WhiteheAd (E. L.) (1957), - Milk drinking by college students. J. amer. diet. Assoc. 33, 1266-1269.

Luzzatto-Fegiz (P.) and Lolli (G.) (1957). - The use of milk and wine in Italy. Quart. J. studies alcohol, 18, 355-381.

Usuelli (F.) (1956). - Considerazioni sulla produzione e sul consumo dei latticini in Italia. Riv. zootec., 29, 345-347.

MunCh-PETERSEN (E.) (1956). - Consumer acceptance of dairy products, J. austral. inst. agric. sci., 22, 255-259.

Alkemade (T.), Van Leeuwen (P.), De Wijn (J. F.) and Zielhuis (R. L.) (1956). Use of milk and cheese by young people in industry, Voeding, 17, 371-382.

Den HARTOG (C.) (1956). - Significance of milk for the Netherlands population. Voeding, 17, 9-11.

Den Hartog (C.) and De HaAs (J.H.) (1956). - Inquiry into the consumption of milk and cheese by rural schoolchildren. Voeding, 17, 12-26.

Van SyCKLE (C.) (1955). - Consumption of fresh and evaporated milk and dry milk solids. J. amer. diet. Assoc., 31, 1228-1231.

MARr (J.W.), Hope (E.B.), Stevenson (J.D.) and Thompson (A.M.) (1955). - Consumption of milk and vitamins by pregnant women. Brit. J. nutrit., 14, i-vii. 